\title{
Chapter 11 \\ Hypotensive Hemostasis in Patients Presenting with Ruptured Aortic Aneurysm
}

\author{
Felice Pecoraro, Bernard Krüger, Johnny Steuer, Neal Cayne, \\ Zoran Rancic, Frank J. Veith, and Mario Lachat
}

\section{Introduction}

In contrast to peripheral vascular injury, which may be controlled with a tourniquet or manual compression to stabilize the patient before definitive repair, in the ruptured intrathoracic and intra-abdominal aorta or its branches, external compression is rarely an alternative. Acute and severe blood loss leads to hypotension and cardiovascular shock, resulting in multisystem organ failure and eventual death. However, aggressive fluid resuscitation in these patients can be detrimental as large-volume infusion may lead to further blood loss, hemodilution [1], dilutional

\section{F. Pecoraro}

Clinic for Cardiovascular Surgery, University Hospital, Zurich, Switzerland

Institute for Cardiovascular Anesthesia, University Hospital, Zurich, Switzerland

B. Krüger

Vascular Surgery Unit, University of Palermo, University Hospital 'P. Giaccone',

Palermo, Italy

J. Steuer

Clinic for Cardiovascular Surgery, University Hospital, Zurich, Switzerland

Department of Surgery, Section for Vascular Surgery, South Hospital, Stockholm, Sweden

N. Cayne

New York University Medical Center, New York, NY, USA

Z. Rancic $\bullet$ M. Lachat $(\bowtie)$

Clinic for Cardiovascular Surgery, University Hospital, Zurich, Switzerland

e-mail: mario.lachat@usz.ch

F.J. Veith

Clinic for Cardiovascular Surgery, University Hospital, Zurich, Switzerland

New York University Medical Center, New York, NY, USA 
coagulopathy, and hypothermia [2]. Moreover, in ruptured abdominal aortic aneurysm (rAAA), massive retroperitoneal bleeding contributes to the development of intra-abdominal hypertension (IAH) and ultimately to abdominal compartment syndrome (ACS) with a high risk of severe complications and death [3]. Finally, massive transfusion of red blood cells, thrombocytes, and/or coagulation factors may be accompanied by severe immunologic reaction and transfusion-related acute lung injury (TRALI) [4]. To avoid these scenarios and/or complications, hypotensive hemostasis is advocated.

From a historical point of view, in 1949 Andresen et al. reported their experience in the management of gastric hemorrhage suggesting that "No transfusion should be given in the first days except for evidence of severe anoxia. Then try 1 or 2 transfusions of 150-200 CC of citrated blood" [5]. This original experience first advised a switch from resuscitation with high amount of fluids to achieve a neophysiological blood pressure to the concept of hypotensive hemostasis, limiting the resuscitation fluids in cases of massive blood loss. In 1981, Akins showed in an observational single-center study that in patients with blunt aortic injury, the use of beta-blockers and antihypertensive therapy permitted stabilizing the patient to allow delayed aortic repair until extracorporeal circulation with the use of a heart-lung machine could be initiated, which was often a necessity in the era preceding the current use of endovascular aneurysm repair (EVAR) [6]. In 1991 Crawford [7] advocated, in patients presenting with rAAA, the use of small volumes of whole blood or crystalloid to maintain blood pressure around $50-70 \mathrm{mmHg}$ until cross-clamping of the aorta was accomplished. A similar fluid restriction scheme was reported in 2002 by Veith and Ohki [8] for EVAR in rAAA. Controlled hypotension using vasodilators to lower the blood pressure to $<100 \mathrm{mmHg}$ was reported first by our group in 2002 and later by Yilmaz et al. [9]. In 2007 Blankensteijn confirmed in a prospective (nonrandomized) study the feasibility of controlled hypotension in candidates for open surgery and endovascular repair. However, to date, there is no prospective randomized controlled trial demonstrating the advantage of hypotensive hemostasis over standard resuscitation management [10].

Resuscitation is generally initiated when aortic rupture is confirmed by ultrasound and/or CTA. In analogy to trauma patients, reducing the time from the initial event to having the bleeding under control in rAAA may increase the chance of survival [11]. Based on a Cochrane meta-analysis, there is no clear evidence on which type of fluid should be employed (crystalloids or colloids) in patients presenting with hemorrhagic shock. However, the lack of outcome differences made the authors advise the use of crystalloids for economic reasons [12]. If fluid administration is not sufficient to restore the arterial blood pressure or undesirable such as in the presence of a large retroperitoneal hematoma, vasopressor drugs may be employed in order to reduce the risk of tissue hypoperfusion. However, the use of vasopressors is justified only transiently and just to reach systolic arterial pressures of 80-90 $\mathrm{mmHg}$ [13]. If cardiac function is depressed, an inotropic agent should be utilized [14]. Control of corporeal temperature is another relevant measure that can influence not only arterial pressure but also acid-base status and coagulation. 
Hypothermia $\left(<35^{\circ}\right)$ is associated with higher mortality and morbidity in patients presenting with hypotension in association with hemorrhagic shock [15]. In 1965 Shaftan et al. reported that "Blood loss from an arterial injury is greatest in quantity and prolonged when fluids or vasopressors are given and least and shortest when either resuscitation is withheld or vasodilators are administrated" [16]. In fact, general anesthesia, necessary for the open approach in rAAA, and the resulting vasoplegia following its induction require a high volume of fluids to achieve an adequate volume balance and blood pressure. This fluid load increases the risk of hemorrhage, hypothermia and coagulation disorders, and abdominal compartment syndrome. Moreover, general anesthesia with the use of myorelaxation decreases the abdominal wall tone and therefore may contribute to the transition from a contained to a frank aortic aneurysm rupture $[17,18]$.

\section{Hypotensive Hemostasis Protocol and Experience at the University Hospital Zurich (USZ)}

The hypotensive hemostasis protocol at USZ is based on permissive hypovolemia and controlled hypotension. Permissive hypovolemia consists in minimizing the administration of fluids (crystalloids and/or colloids), whereas controlled hypotension implies active lowering of the blood pressure with vasodilators and/or betablockers in normotensive or hypertensive patients to a target systolic blood pressure $<90 \mathrm{mmHg}$. In such patients, fluid infusion is restricted to a minimum just to keep the intravenous lines open.

In patients presenting with low blood pressure but who are otherwise hemodynamically stable, some fluids $(100-500 \mathrm{ml})$ may be administered to maintain a target systolic blood pressure of $\geq 70 \mathrm{mmHg}$. We define "stable" as a systolic blood pressure of any value that does not require an increase in the amount of fluids or vasoactive pressors/dilators to remain constant over a longer period of time ( $>5 \mathrm{~min}$ ). In patients who are critically hypotensive or who are unstable or who get unconscious, a bolus of $250 \mathrm{ml}$ of fluid (colloid or crystalloid or blood) may be infused. In addition, vasoactive pressors may eventually be required to restore and/or maintain the systolic blood pressure around $70 \mathrm{mmHg}$. Even under such low blood pressure circumstances, conversion to general anesthesia is not necessary as long as the patient maintains intact airway reflexes. Transfusion of blood cells, coagulation factors, and platelets is based on the respective guidelines. Briefly, hematocrit is maintained over $24 \%$, and transfusion of coagulation factors and/or platelets is based on blood samples and rotational thromboelastometry (ROTEM).

The pressure-lowering effects of vasodilators are often more pronounced in patients with hypovolemia. Drugs must therefore be titrated carefully to the desired effect. Short-acting vasoactive drugs have a limited effect with regard to time. In case of inadvertent over-dosage or intentional termination of the permissive hypotension after achieving sufficient hemostasis, the hypotensive effect of the drugs will fade rapidly. 


\section{Vasoactive Drugs}

Esmolol, a short-acting and selective adrenergic $\beta 1$-receptor antagonist, decreases heart rate and contractility, is metabolized by red blood cell esterases, and has an elimination half-life of approximately $9 \mathrm{~min}$. It can be titrated to effect by repetitive i.v. bolus doses of $100 \mu \mathrm{g} / \mathrm{kg}$ up to a dose of $500 \mu \mathrm{g} / \mathrm{kg}$ followed by continuous i.v. infusion up to $125 \mu \mathrm{g} / \mathrm{kg} / \mathrm{min}$. The target range of the heart rate is $60-80 \mathrm{bpm}$.

Glyceryl trinitrate (nitroglycerin), a vasodilator with more prominent effects on peripheral veins than on arteries, decreases the preload of the heart by pooling of blood in the venous system. The elimination half-life is 2-3 min. Onset of action is immediate by relaxation of smooth muscle cells following an increase of intracellular concentrations of cyclic guanosine monophosphate (cGMP). Titration to effect is done by repetitive i.v. bolus doses of $25 \mu \mathrm{g}$ up to $75-100 \mu \mathrm{g} / \mathrm{min}$ followed by a continuous i.v. infusion of up to $300 \mu \mathrm{g} / \mathrm{min}$. Reflex tachycardia is a physiological response to decreased preload and can be counteracted by esmolol i.v.

After control of bleeding has been achieved in the operating room, all pressurelowering drugs are discontinued, and hypovolemia is corrected as necessary. Appropriate fluid resuscitation is the base for vasoactive drugs to sufficiently raise systemic blood pressure to the desired level. Norepinephrine, acting primarily as a vasopressor, is the first choice, followed by epinephrine as second choice in case of profound shock and/or in order to enhance the cardiac contractility.

Norepinephrine (noradrenaline), a stimulator of both adrenergic $\alpha 1 / 2$-receptors and $\beta 1$-receptors, raises the systemic blood pressure primarily by arterial vasoconstriction to a lower extent by its positive inotropic and chronotropic effects. It has an immediate onset of action and a half-life of 2-3 min. An i.v. bolus of $0.1 \mu \mathrm{g} / \mathrm{kg}$ is repetitively given followed by a continuous i.v. infusion of $0.01-0.3 \mu \mathrm{g} / \mathrm{kg} / \mathrm{min}$ (0.8-24 $\mu \mathrm{g} / \mathrm{min}$ for an $80 \mathrm{~kg}$ patient).

In cases of persisting clinical and laboratory signs of arterial hypoperfusion after appropriate correction of hypovolemia and vasopressor support with norepinephrine $0.3 \mu \mathrm{g} / \mathrm{kg} / \mathrm{min}$, impaired cardiac function may be the underlying cause, and epinephrine should be considered to improve cardiac inotropy.

Epinephrine (adrenaline) stimulates adrenergic $\beta 1 / 2$-receptors with a resulting increase in cardiac chrono- and inotropy at lower dosage $(0.01-0.1 \mu \mathrm{g} / \mathrm{kg} / \mathrm{min}$ i.v.) and adrenergic $\alpha 1 / 2$-receptors with progressive peripheral arterial vasoconstriction at higher dosage $(0.1-0.3 \mu \mathrm{g} / \mathrm{kg} / \mathrm{min}$ i.v.). It has an immediate onset of action and a half-life of 2-3 min. If epinephrine is needed as a bolus (0.1-0.2 $\mu \mathrm{g} / \mathrm{kg}$ i.v.), cardiopulmonary resuscitation is usually imminent.

Hypovolemia must be repeatedly ruled out or corrected if high doses of noradrenaline are infused, particularly if combined with epinephrine, since hypovolemic vasoconstriction may cause profound tissue ischemia and necrosis. Simple use of a central line pressure or wedge pressure measurement from a Swan-Ganz catheter can help determine if the patient needs additional volume or pressor medications (Table 11.1). 


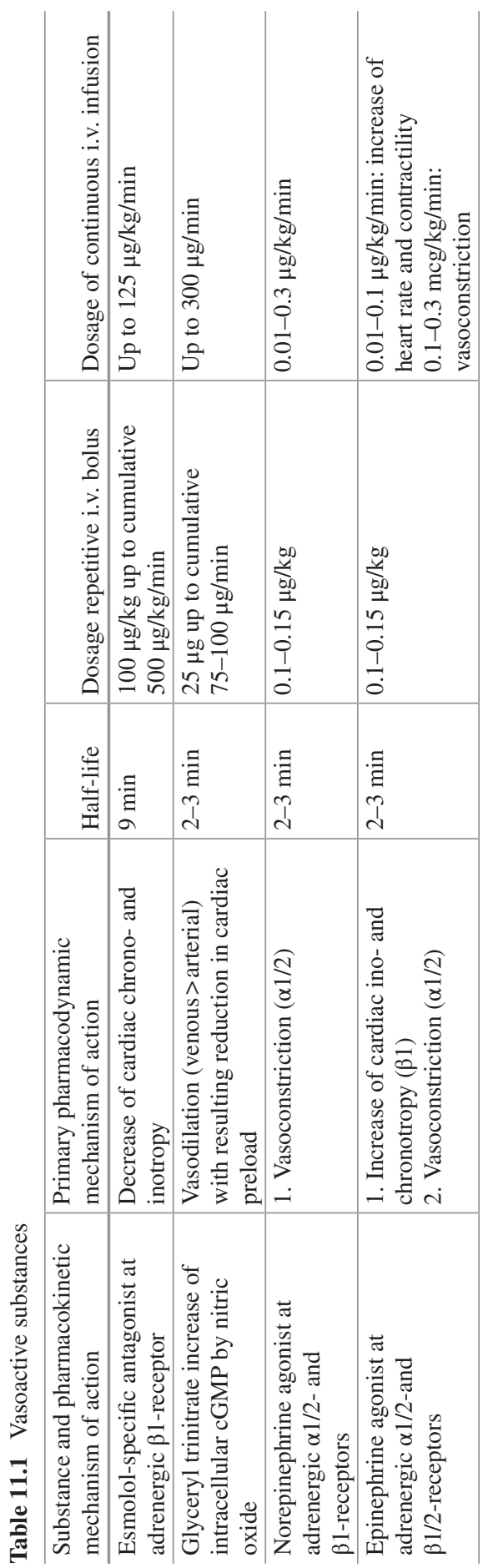




\section{Intra-aortic Balloon}

Endoclamping of the aorta with a large-size compliant balloon such as Reliant ${ }^{\circledR}$ (Medtronic) or the Coda (Cook) has been shown to be useful in patients with unstable hemodynamics and/or those patients unresponsive to fluids and catecholamines (Fig. 11.1). Endoclamping can be used for both the endovascular and the surgical approach. In stable low-pressure patients, especially when a large retroperitoneal hematoma has been identified preoperatively, it can also be used during the EVAR procedure to avoid further volume increase of the hematoma. The balloon can be placed under local anesthesia, utilizing a long (preferably $\geq 40 \mathrm{~cm}$ ) sheath for prevention of caudal migration during and after inflation. To avoid balloon-related complications, the inflation site should be free of major atherosclerotic aortic disease or aneurysm. Moreover, as the balloon is placed superior to the major branches of the abdominal aorta, the duration of the balloon inflation should be kept as short as possible, since inflation brings about temporary renovisceral ischemia.

\section{Discussion}

Based on the early positive experience and subsequent systematic follow-up of the patients at our center, we have routinely applied the principles of hypotensive hemostasis for nearly 20 years. The feasibility of EVAR in rAAA using hypotensive hemostasis, including permissive hypovolemia, controlled hypotension, and local anesthesia, was reported by our group in 2001 [19]. In this series of 21 patients, restricted volumes of fluids and erythrocyte transfusions were enough to maintain hemodynamic stability prior to the completion of EVAR (Fig. 11.2).

Fig. 11.1 Intraoperative angiogram. After placing a guide wire, an aortic balloon is delivered and inflated suprarenal (here supraceliac) to achieve proximal hemorrhage control

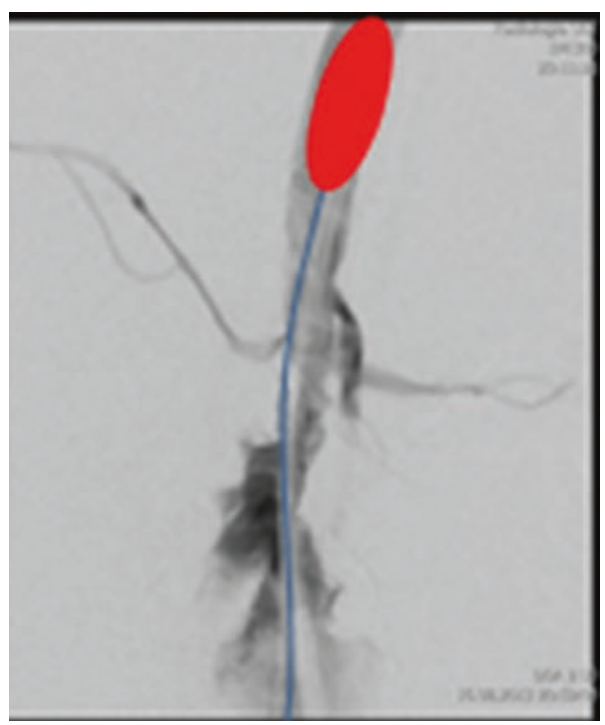


Total volume (ml) infused: Fluids+ Ec+ FFP+Tc

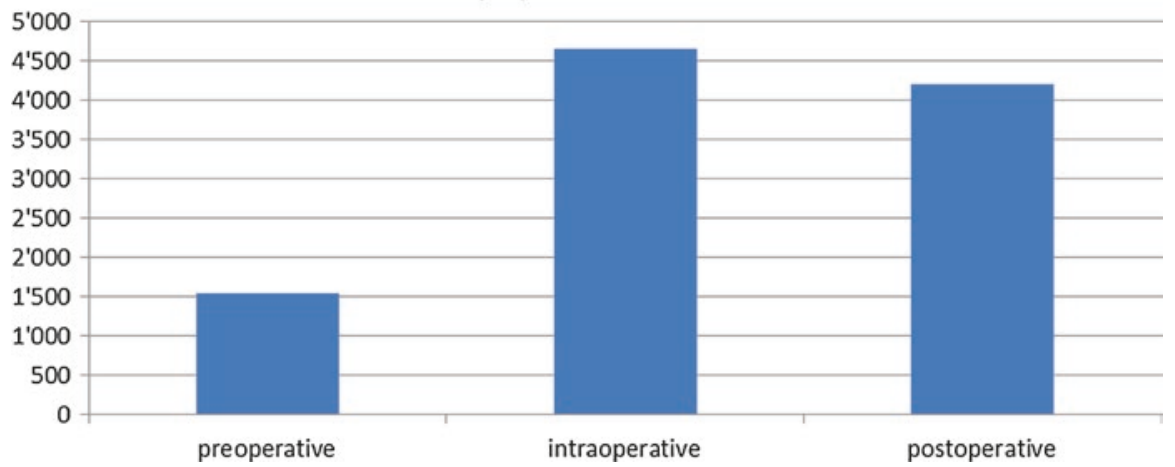

Fig. 11.2 Total amount of fluids and transfusions given preoperatively, intraoperatively, and postoperatively. Fluids crystalloids and/or colloids, Ec erythrocytes, FFP fresh frozen plasma, Tc thrombocytes, (Modified from Lachet et al. [19])

Generally, most patients remain stable with preserved mental function at a systolic blood pressure of $70-90 \mathrm{mmHg}$. To achieve stable hemodynamics at this blood pressure level, very limited amounts of fluids and vasoactive pressors are necessary during EVAR under local anesthesia. As a first step, analgesia is optimized to decrease the sympathomimetic activity. Low dosages of opioids are used and titrated to the desired effect. In patients undergoing interventions under local anesthesia, special care must be taken not to abolish consciousness and risk compromising spontaneous breathing and the protective airway reflexes. Advantages of local anesthesia include the preservation of sympathetic tone, yielding improved hemodynamic stability, preservation of muscle tone, and thereby the possibility to better contain the bleeding, and, in addition, any procedure under local anesthesia offers the best neurological monitoring. The main disadvantages are related to eventual acute loss of consciousness and an uncontrolled airway, suboptimal pain control, and the patient not lying still. Conversely, general anesthesia has the advantages of a controlled airway and optimal pain control. However, by using general anesthesia, most of the advantages of local anesthesia are lost, and it is more time-consuming when compared to local. If general anesthesia is required for an open operation, the patient should be fully prepped and draped prior to induction, and, ideally, an aortic occlusion balloon should first be placed under local anesthesia so that it can be inflated in cases of severe hypotension at any point prior to or during the procedure.

The use of hypotensive hemostasis was advocated by the clinical practice guidelines of the European Society for Vascular Surgery as it "might have a beneficial effect on the survival in cases of abdominal aortic aneurysm rupture." To maintain blood pressure in a range of 50-100 $\mathrm{mmHg}$ was recommended [20]. Limitations of hypotensive hemostasis are related mainly to brain, heart, and kidney perfusion. The brain perfusion is self-regulated within the systolic blood pressure range of $50-100 \mathrm{mmHg}$. The risk of cerebral hypoperfusion and thus ischemic stroke especially in patients with concomitant cerebrovascular disease should always be 
acknowledged. If the operation is conducted under local anesthesia, initial signs of cerebral hypoperfusion can be realized early, and cerebral perfusion is usually satisfactory if the patient responds adequately to neurological stimuli and is communicating verbally. Also, coronary perfusion may be at risk with low blood pressure. In patients with known coronary artery disease undergoing the procedure under local anesthesia, electrocardiographic and clinical signs of myocardial ischemia should be analyzed, whereas in patients operated under general anesthesia, transesophageal echocardiography should be considered. The kidneys generally have a fairly good tolerance of temporary ischemia. However, concurrent insults, such as contrast medium injection, increase the risk of renal injury. Urine output is a valid tool in the monitoring of the renal function.

In summary, aggressive fluid resuscitation in aorta rupture patients can be detrimental, as large-volume infusion can lead to further blood loss and hemodilution, coagulopathy, and hypothermia. Moreover, massive retroperitoneal bleeding may result in abdominal compartment syndrome with a high risk of further severe complications and death. Finally, extensive transfusion of blood cells, thrombocytes, and/or coagulation factors may be accompanied by transfusion-related acute lung injury. To avoid such scenarios and/or complications, hypotensive hemostasis, including permissive hypovolemia and controlled hypotension, is advocated.

\section{References}

1. Morrison CA, Carrick MM, Norman MA, Scott BG, Welsh FJ, Tsai P, et al. Hypotensive resuscitation strategy reduces transfusion requirements and severe postoperative coagulopathy in trauma patients with hemorrhagic shock: preliminary results of a randomized controlled trial. J Trauma. 2011;70(3):652-63.

2. Bougle A, Harrois A, Duranteau J. Resuscitative strategies in traumatic hemorrhagic shock. Ann Intensive Care. 2013;3(1):1.

3. Mayer D, Rancic Z, Veith FJ, Pecoraro F, Pfammatter T, Lachat M. How to diagnose and treat abdominal compartment syndrome after endovascular and open repair of ruptured abdominal aortic aneurysms. J Cardiovasc Surg. 2014;55(2):179-92.

4. Vlaar AP, Hofstra JJ, Determann RM, Veelo DP, Paulus F, Kulik W, et al. The incidence, risk factors, and outcome of transfusion-related acute lung injury in a cohort of cardiac surgery patients: a prospective nested case-control study. Blood. 2011;117(16):4218-25.

5. Andresen AF. Management of gastric hemorrhage. Modern Med. 1949;17(20):72-5.

6. Akins CW, Buckley MJ, Daggett W, McIlduff JB, Austen WG. Acute traumatic disruption of the thoracic aorta: a ten-year experience. Ann Thorac Surg. 1981;31(4):305-9.

7. Crawford ES. Ruptured abdominal aortic aneurysm. J Vasc Surg. 1991;13(2):348-50.

8. Veith FJ, Ohki T. Endovascular approaches to ruptured infrarenal aorto-iliac aneurysms. J Cardiovasc Surg. 2002;43(3):369-78.

9. Yilmaz N, Peppelenbosch N, Cuypers PW, Tielbeek AV, Duijm LE, Buth J. Emergency treatment of symptomatic or ruptured abdominal aortic aneurysms: the role of endovascular repair. J Endovasc Ther. An Official Journal of the International Society of Endovascular Specialists. 2002;9(4):449-57.

10. van der Vliet JA, van Aalst DL, Schultze Kool LJ, Wever JJ, Blankensteijn JD. Hypotensive hemostatis (permissive hypotension) for ruptured abdominal aortic aneurysm: are we really in control? Vascular. 2007;15(4):197-200. 
11. Cothren CC, Moore EE, Hedegaard HB, Meng K. Epidemiology of urban trauma deaths: a comprehensive reassessment 10 years later. World J Surg. 2007;31(7):1507-11.

12. Perel P, Roberts I. Colloids versus crystalloids for fluid resuscitation in critically ill patients. Cochrane Database Syst Rev. 2011(3):CD000567

13. Dellinger RP, Levy MM, Carlet JM, Bion J, Parker MM, Jaeschke R, et al. Surviving Sepsis Campaign: international guidelines for management of severe sepsis and septic shock: 2008. Crit Care Med. 2008;36(1):296-327.

14. Kaye P, O'Sullivan I. Myocardial contusion: emergency investigation and diagnosis. Emerg Med J. 2002;19(1):8-10.

15. Hoey BA, Schwab CW. Damage control surgery. Scand J Surg. SJS Official organ for the Finnish Surgical Society and the Scandinavian Surgical Society. 2002;91(1):92-103.

16. Shaftan GW, Chiu CJ, Dennis C, Harris B. Fundamentals of physiologic control of arterial hemorrhage. Surgery. 1965;58(5):851-6.

17. Kozek-Langenecker SA, Afshari A, Albaladejo P, Santullano CA, De Robertis E, Filipescu DC, et al. Management of severe perioperative bleeding: guidelines from the European Society of Anaesthesiology. Eur J Anaesthesiol. 2013;30(6):270-382.

18. Bettex DA, Lachat M, Pfammatter T, Schmidlin D, Turina MI, Schmid ER. To compare general, epidural and local anaesthesia for endovascular aneurysm repair (EVAR). Eur J Vasc Endovasc Surg. The Official Journal of the European Society for Vascular Surgery. 2001;21(2):179-84.

19. Lachat ML, Pfammatter T, Witzke HJ, Bettex D, Kunzli A, Wolfensberger U, et al. Endovascular repair with bifurcated stent-grafts under local anaesthesia to improve outcome of ruptured aortoiliac aneurysms. Eur J Vasc Endovasc Surg. The Official Journal of the European Society for Vascular Surgery. 2002;23(6):528-36.

20. Moll FL, Powell JT, Fraedrich G, Verzini F, Haulon S, Waltham M, et al. Management of abdominal aortic aneurysms clinical practice guidelines of the European society for vascular surgery. Eur J Vasc Endovasc Surg. The Official Journal of the European Society for Vascular Surgery. 2011;41 Suppl 1:S1-58. 